\title{
Overexpression of SOX18 promotes prostate cancer progression via the regulation of TCF1, c-Myc, cyclin D1 and MMP-7
}

\author{
HUAQI YIN $^{1 *}$, ZHENGZUO SHENG $^{1 *}$, XIAOWEI ZHANG ${ }^{1}$, YIQING DU $^{1}$, CAIPENG QIN $^{2}$, HUIXIN LIU $^{1}$, \\ YAOJUN DUN ${ }^{1}$, QI WANG ${ }^{1}$, CHENGYUE JIN $^{1}$, YANHUI ZHAO ${ }^{3}$ and TAO XU ${ }^{1}$ \\ ${ }^{1}$ Department of Urology, Peking University People's Hospital, Beijing; ${ }^{2}$ Department of Urology, Peking University \\ International Hospital, Beijing; ${ }^{3}$ Department of Urology, Central Hospital of Qingdao City, Qingdao, P.R. China
}

Received June 16, 2016; Accepted July 28, 2016

DOI: $10.3892 /$ or.2016.5288

\begin{abstract}
Sex determining region Y (SRY)-box 18 (SOX18) gene encodes transcription factors that have been recently confirmed to be overexpressed in various human types of cancer and maintain the malignant behavior of cancer cells. However, the role and its potential function in prostate cancer $(\mathrm{PCa})$ has not been demonstrated and the mechanisms of SOX18 involved in tumor progression remain largely unclear. In the present study, the expression of SOX18 was analyzed in $98 \mathrm{PCa}$ and 81 adjacent non-tumor tissues using immunohistochemistry. The data showed that SOX18 was overexpressed in 72 of $98(73.5 \%)$ PCa tissues compared with that in 28 of 81 (34.6\%) non-tumor tissues. In addition, the expression of SOX18 was related with the clinical features of patients with $\mathrm{PCa}$. To explore the potential role of SOX18 in PCa cells, Cell Counting Kit-8 (CCK-8), migration, invasion and xenograft assays were performed. Our data showed that knockdown of SOX18 decreased the proliferation, migration and invasion of PCa cells in vitro, in addition to the tumor growth in vivo. Markedly, SOX18 knockdown caused the decreased expression of TCF1, c-Myc, cyclin D1 and MMP-7. In conclusion, SOX18 was overexpressed in PCa and may regulate the malignant capacity of cells via the upregulation of TCF1, c-Myc, cyclin D1 and MMP-7.
\end{abstract}

\section{Introduction}

Prostate cancer (PCa), a clinically heterogeneous multifocal disease, remains a major health concern in men, with an estimated 180,890 new cases and 26,120 deaths in the US in

Correspondence to: $\mathrm{Dr}$ Tao Xu, Department of Urology, Peking University People's Hospital, 11 Xi Zhi Men South Street, 100044 Beijing, P.R. China

E-mail:xutao@medmail.com.cn

*Contributed equally

Abbreviations: SOX18, sex determining region Y (SRY)-box 18; MMP-7, matrix metalloproteinase-7

Key words: prostate cancer, transcription factor, SOX18, tumor progression, TCF1, c-Myc, cyclin D1, MMP-7
2016 (1). Although strategies such as lifestyle modifications offer opportunities to reduce the risk of $\mathrm{PCa}$, the incidence of $\mathrm{PCa}$ has continued to increase in recent years (2). To date, the early detection of PCa by PSA screening has been controversial for many years. Therefore, optimization for prostate-specific antigen (PSA) screening strategies and finding new biomarkers may minimize overdiagnosis related with PSA screening and improve the detection rate of $\mathrm{PCa}$. Even after removal, $>20 \%$ of patients suffer a recurrence, mostly detected by a rise in the serum level of PSA (3). Facing this global health issue, the identification of new biomarkers and potential oncogenes involved in PCa may provide more sophisticated ways for the early diagnosis and further treatment.

The SOX family, consisting of a number of transcription factors that contain a highly conserved high-mobility group (HMG) DNA-binding domain, plays critical roles in regulating cell fate decisions during development (4). The SOX family is comprised of $>20$ members identified by homology-based analysis of the HMG DNA-binding domain (5). In recent years, accumulating research has confirmed the link between SOX genes and various human diseases including cancer (6). The expression of $S O X$ genes was found to differ in various types of cancer, and their functions in cancer development are also heterogeneous (7). For instance, SOX2 was found to be overexpressed in adenoid cystic carcinoma (ACC) and is associated with the poor prognosis in patients with ACC (8). However, overexpression of SOX 2 was found to indicate a favorable prognosis in patients with non-small cell lung cancer (9). SOX10, SOX8 and SOX9 are overexpressed in hepatocellular carcinoma (HCC) and SOX10 is an oncogene promoting the progression of HCC (10). In the case of the sex determining region Y (SRY)-box 18 (SOX18), it has been determined that it participates in the process of angiogenesis and lymphangiogenesis, and loss of SOX18 is responsible for hypotrichosis-lymphodema-teleangiectasia syndrome (11-13). A growing number of studies suggest that SOX18 is overexpressed in various types of tumors and may play an important role in tumor occurrence and progression (14). It has been documented that SOX18 is overexpressed in HCC, ovarian and non-small cell lung cancer and ductal breast and cervical carcinoma compared with normal tissues and is related with a poor prognosis in patients with these types of cancer (15-18). Furthermore, SOX18 may regulate the 
progression of tumors via the activation of its downstream transcription factors such as MMP-7 and endothelial-specific claudin-5 (19,20). However, the expression level and biological function of SOX18 in PCa remain unclear, and the potential mechanisms involved still need to be addressed.

In the present study, we observed a frequent overexpression of SOX18 in PCa tissues. Silencing of SOX18 significantly impaired the proliferation, migration and invasion ability of PCa cells in vitro and also supressed tumor growth in vivo. Statistical analysis demonstrated that a high expression of SOX18 was correlated with the poor clinical features of patients with PCa. Markedly, knockdown of SOX18 in PC3 cells induced decreased expression of TCF1, c-Myc, cyclin D1 and MMP-7, which establishes it as an oncogene.

\section{Materials and methods}

Tissue microarray. A tissue microarray with 179 spots of human PCa and adjacent normal tissues, or benign hyperplasia (BPH) tissues (98 PCa and 81 normal/BPH tissues) was obtained from Shanghai Outdo Biotech (Shanghai, China).

Cell lines, siRNAs and lentivirus. Three PCa cell lines PC3, DU145 and LNcaP were directly obtained from the American Type Culture Collection (ATCC; Manassas, VA, USA) for no more than 6 months. Cells were cultured in RPMI-1640 (HyClone, Logan, UT, USA) medium supplemented with $10 \%$ fetal bovine serum (FBS) (Gibco, Sydney, Australia) and 1\% penicillin/streptomycin (Gibco, Grand Island, NY, USA), and incubated at $37^{\circ} \mathrm{C}$ in a humidified atmosphere with $5 \% \mathrm{CO}_{2}$.

Small interfering RNAs (siRNA) targeting SOX18 were synthesized by GenePharma (Suzhou, China). The following sequences were used: siRNA1, 5'-GGGUUACAUUUUUGAA GCATT-3' (sense) and 5'-UGCUUCAAAAAUGUAACCC TT-3' (antisense); siRNA2, 5'-CUCUCUCAUACGCGUGUAU TT-3' (sense) and 5'-AUACACGCGUAUGAGAGAGTT-3' (antisense); and negative control (siNC) 5'-UUCUCCGAACG UGUCACGUTT-3' (sense) and 5'-ACGUGACACGUUCGGA GAATT-3' (antisense). The siRNAs were transfected into PC3 and DU145 cells using Lipofectamine 3000 Transfection kit (Invitrogen, Carlsbad, CA, USA) according to the manufacturer's instructions. The efficiency of SOX18 silencing was determined using western blotting.

Lentiviruses carrying the silencing sequence (LV-SOX18) and the negative control sequence (LV-NC) were packaged by the Shanghai GeneChem Co., Ltd. (Shanghai, China). The silencing sequence was the same as the siRNA2 and the control sequence as the siNC. The PC3 and DU145 cell lines were infected with the lentiviruses at a multiplicity of infection (MOI) of 100 for $24 \mathrm{~h}$. Then, puromycin (Calbiochem, La Jolla, CA, USA) was used to screen the stably infected cells. The efficiency of infection was assessed by western blotting.

$R T-P C R$. The preparation of total RNA and the performance of RT-PCR were as previously described (21). The primers used were as follows: SOX18 (forward, 5'-CGCGTGTATGTT TGGTTC-3' and reverse, 5'-ATGTAACCCTGGCAACTC-3'); and GAPDH (forward, 5'-CACCCACTCCTCCACCTTTG-3' and reverse, 5'-CCACCACCCTGTTGCTGTAG-3').
Immunohistochemical analysis. After being dewaxed, rehydrated and blocked with $30 \%$ normal goat serum for $30 \mathrm{~min}$, the microarray was incubated with rabbit monoclonal antibody against SOX18 (1:100, Abcam, Cambridge, MA, USA) in a moist chamber at $4^{\circ} \mathrm{C}$ overnight. Immunodetection was conducted using the Envision ABC kit (Gene Tech Co., Ltd. Shanghai, China). After staining with hematoxylin, the microarray was dehydrated and mounted. The intensity and extent of SOX18 staining were evaluated by two experienced pathologists without the clinical data, respectively. The method for calculating the score of SOX18 staining was as follows: the extent of staining in an $\mathrm{x} 200$ field was scored as $0,0 \%$; $1,1-25 \% ; 2,26-50 \% ; 3,51-100 \%$. The intensity of staining was scored as 0 , no signal; 1 , light brown; 2 , brown; 3 , dark brown. The final score of each field was the average obtained from the two pathologists from multiplying the extent score by the intent score. The scores of SOX18 staining were categorized as follows: low expression (-/+), when scores were 0-1 (-) and 2-3 (+); high expression $(++/+++)$, when scores were 4-6 (++) and 7-9 (+++). All evaluations were performed using a Leica DM4000 M microscope.

Western blotting. The cells were lysed on ice in RIPA buffer (Solarbio, Beijing, China) supplemented with a $1 \%$ protease inhibitor cocktail (Thermo Scientific, Rockford, IL, USA). The protein concentration was assessed using BCA protein assays (Solarbio, Beijing, China). Equal amounts of proteins were separated using $10 \%$ SDS-PAGE gels, and were then transferred onto nitrocellulose membranes (Millipore, Bedford, MA, USA). The membranes were blocked in rapid blocking liquid (Promoton, Shanghai, China) for $10 \mathrm{~min}$ and incubated with a primary antibody at $4^{\circ} \mathrm{C}$ overnight. After being incubated with the secondary antibody for $1 \mathrm{~h}$ at room temperature, the immunoreactive bands were detected by Chemiluminescent and Fluorescent Imaging System (Sagecreation, Hangzhou, China). The primary antibodies were SOX18 rabbit monoclonal antibody $(1: 1,000$; Abcam), GAPDH rabbit monoclonal antibody $(1: 3,000)$ and $\beta$-catenin/TCF1/c-Myc/cyclinD1/c-Jun/MMP-7/MMP-2/MMP-9 rabbit monoclonal antibodies [1:1,000; all from Cell Signaling Technology (CST), Inc., Danvers, MA, USA]. The secondary antibodies were goat anti-rabbit IgG/HRP (Bioss, Beijing, China) and the intensity of the target proteins was normalized to the intensity of GAPDH.

Cell Counting Kit-8 (CCK-8) assays. After transfection for $24 \mathrm{~h}, 1,500$ cells were seeded into 96-well plates in $100 \mu \mathrm{l}$ conditioned medium with $10 \%$ FBS. For quantitation of cell proliferation, CCK-8 assays were performed. Briefly, $10 \mu \mathrm{l}$ of CCK-8 reagent (Dojindo, Kunamoto, Japan) was added to each well and incubated at $37^{\circ} \mathrm{C}$, and then the absorbance of each well at $450 \mathrm{~nm}$ was assessed after $1.5 \mathrm{~h}$. Each experiment was performed in triplicate.

Cell migration and invasion assays. Twenty-four hours after transfection with siRNAs, the PC3 and DU145 cells were starved for $6 \mathrm{~h}$. Then, $5 \times 10^{4}$ cells in $200 \mu \mathrm{l}$ serum-free media from each group were added into the upper chamber of a 24-well Transwell or invasion chamber (Corning, Corning, New York, USA) with a polycarbonate filter ( $8-\mu \mathrm{m}$ pore size). 
The bottom chamber contained the conditioned medium with $10 \%$ FBS. After a 24-h incubation, the non-migrated or noninvaded cells in the upper chamber were scraped off using a cotton swab, and the migrated or invaded cells on the bottom were fixed with methanol and stained with hematoxylin. The number of cells was counted in five randomly chosen fields (magnification, x100). Each experiment was performed in triplicate, and the results were obtained from three individual experiments.

Tumor xenograft model in nude mice. All protocols for the animal experiments were approved by the Peking University Institutional Animal Care and Use Committee. Twenty-four female BALB/c nude mice (4-6 weeks old, weighing 18-22 g) were obtained from Peking University Animal Center, and were randomly divided into 4 groups for tumor xenografts as LV-SOX18 PC3, LV-NC PC3, LV-SOX18 DU145 and LV-NC DU145 groups. Cells $\left(5 \times 10^{6}\right)$ in $80 \mu 1$ PBS were subcutaneously injected into the left and right flanks of nude mice for each time-point (day 0). The mice were raised in a germ-free environment in the animal facility. The tumor diameter was measured every 5 days, and the tumor volume was calculated by: length $\mathrm{x}$ width ${ }^{2} \mathrm{x} 0.5$. After 5 weeks, the mice were sacrificed by carbon dioxide narcosis (day 35), and then the tumors were measured, weighed and photographed.

Statistical analyses. Data are expressed as the mean \pm SEM. Statistical analysis was performed using a rank-sum test and a Student's t-test. Values of $\mathrm{P}<0.05$ were considered as statistically significant differences. All statistical evaluations were carried out by SPSS 17.0 (SPSS, Inc., Chicago, IL, USA).

\section{Results}

SOX18 is overexpressed in PCa tissues. To investigate the expression of SOX18 in PCa and adjacent non-tumor tissues, we measured the SOX18 protein level in a tissue microarray by immunohistochemistry. Compared to the adjacent non-tumor tissues $(28 / 81,34.6 \%)$, high expression of SOX18 was found in 72 of the $98(73.5 \%)$ PCa tissues, and was frequently located in the nuclei of the cells (Fig. 1A). SOX18 was also confirmed in prostate cancer cell lines by western blotting and RT-PCR analyses (Fig. 1B and C).

Expression of SOX18 is significantly correlated with the clinical features of the patients with PCa. According to the results obtained from immunohistochemistry, we analyzed whether the expression of SOX18 was correlated with the clinical features of the PCa cases. The rate of high SOX18 staining in the tissues with histological grades III-IV $(25 / 30,83.3 \%)$ was higher than that in the tissues of grades I-II $(47 / 68,69.1 \%)$. The difference between the cases with a high and low histological grade was significant $(\mathrm{P}<0.05)$. When comparing the positive frequency between a higher Gleason $(\geq 8)$ and lower scores $(\leq 7)$, we found that the high SOX18 expression rate of cases with a higher Gleason score was $88.2 \%(30 / 34)$, which had a higher frequency than cases with a lower score $(65.6 \%, 42 / 64)$ $(\mathrm{P}<0.05)$. More importantly, expression of SOX18 was significantly related to the clinical stage of $\mathrm{PCa}$. A strong positive rate was observed in $77.4 \%$ (24/31) of the tissues with stages III-IV,
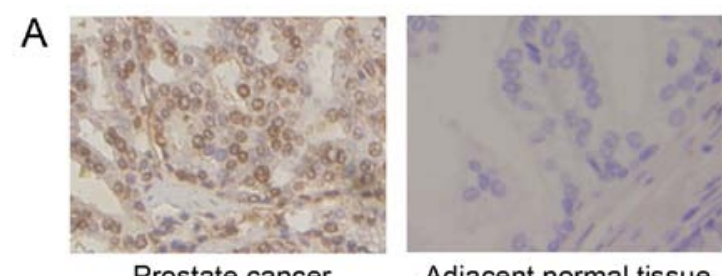

Prostate cancer

Adjacent normal tissue

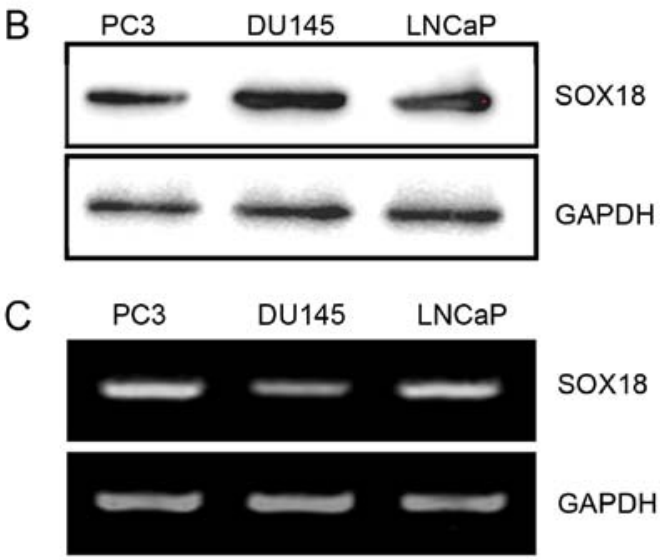

Figure 1. SOX18 is expressed in prostate cancer cells. (A) Overexpression in prostate cancer tissues and negative expression in adjacent normal prostate tissues. (B) SOX18 expression in prostate cancer cell lines by western blotting. (C) SOX18 expression in prostate cancer cell lines by RT-PCR.

Table I. Clinicopathological variables and evaluation of SOX18 immunostaining in prostate cancer tissues.

\begin{tabular}{|c|c|c|c|c|c|c|}
\hline \multirow[b]{2}{*}{ Classification } & \multirow{2}{*}{$\begin{array}{c}\text { No. of } \\
\text { pts. }\end{array}$} & \multicolumn{4}{|c|}{$\begin{array}{l}\text { Scores for SOX18 } \\
\text { staining }\end{array}$} & \multirow[b]{2}{*}{ P-valuc } \\
\hline & & $(-)$ & $(+)$ & $(++)$ & $(+++)$ & \\
\hline Type & & & & & & 0.000 \\
\hline Non-tumor & 81 & 20 & 33 & 24 & 4 & \\
\hline $\mathrm{PCa}$ & 98 & 9 & 17 & 40 & 32 & \\
\hline Age (years) & & & & & & 0.762 \\
\hline$>70$ & 58 & 5 & 12 & 22 & 19 & \\
\hline$\leq 70$ & 40 & 4 & 5 & 18 & 13 & \\
\hline Clinical stage & & & & & & 0.048 \\
\hline I-II & 67 & 6 & 13 & 32 & 16 & \\
\hline III-IV & 31 & 3 & 4 & 8 & 16 & \\
\hline Histological grade & & & & & & 0.010 \\
\hline I-II & 68 & 7 & 14 & 31 & 16 & \\
\hline III-IV & 30 & 2 & 3 & 9 & 16 & \\
\hline Gleason score & & & & & & 0.005 \\
\hline$\leq 7$ & 64 & 6 & 16 & 27 & 15 & \\
\hline$\geq 8$ & 34 & 3 & 1 & 13 & 17 & \\
\hline
\end{tabular}

$\mathrm{PCa}$, prostate cancer; pts., patients.

while the strong positive rate was $71.6 \%(48 / 67)$ in tissues with stages I-II $(\mathrm{P}<0.05)$. However, no relationship between SOX18 expression and age was observed $(\mathrm{P}=0.762)$ (Table I). 
A

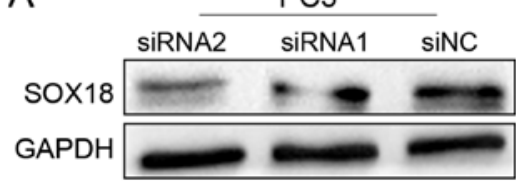

B

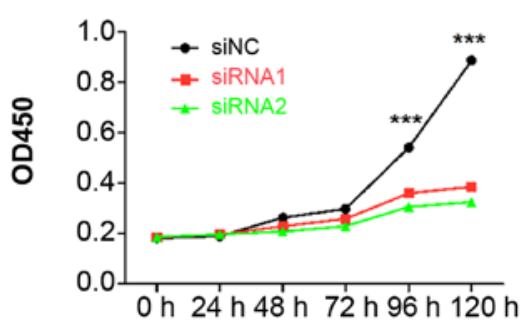

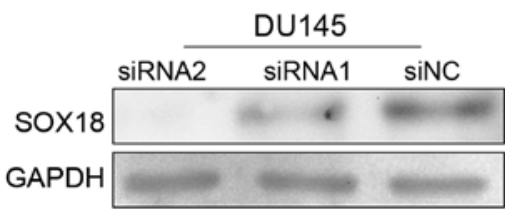

DU145

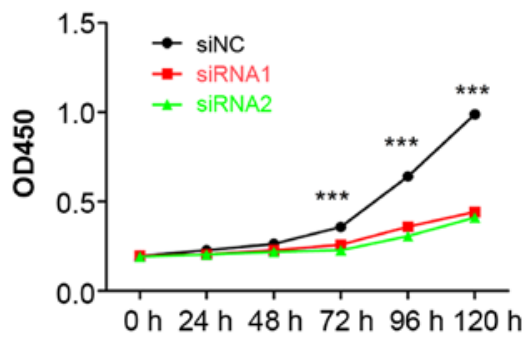

Figure 2. Knockdown of SOX18 significantly decreases the proliferation of prostate cancer cells. (A) The efficiency of SOX18 silencing in PC3 and DU145 cells was detected by western blotting after transfection with siRNAs. (B) Knockdown of the expression of SOX18 inhibited the proliferation of PC3 and DU145 cells $\left({ }^{* * *} \mathrm{P}<0.001\right)$.
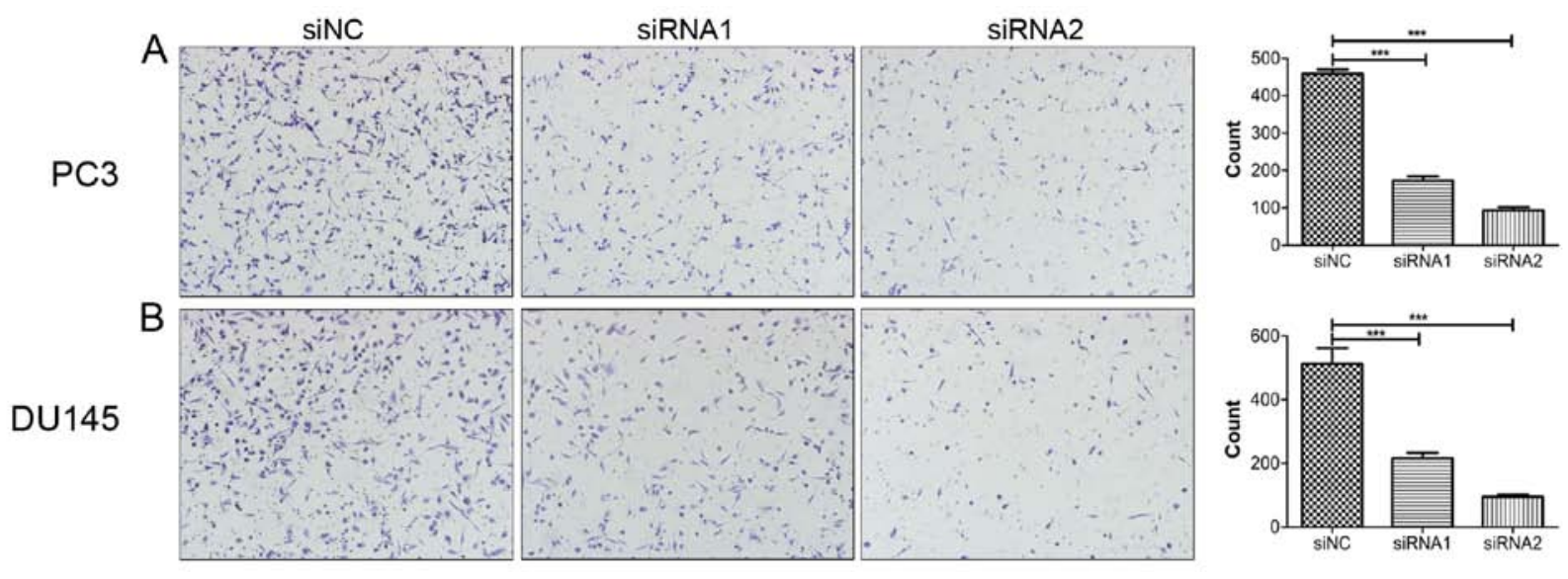

Figure 3. Knockdown of SOX18 significantly decreases the migration of prostate cancer cells. The numbers of migrated (A) PC3 and (B) DU145 cells were decreased in the siRNA groups compared with those in the siNC groups $\left({ }^{* * * *} \mathrm{P}<0.001\right)$.

Knockdown of SOX18 significantly impacts the proliferation of both PC 3 and DU145 cells. To explore the potential role of SOX18 in PCa cell proliferation, we knocked down the expression of SOX18 in both PC3 and DU145 cell lines using two siRNAs, and analyzed the cell proliferation ability using CCK-8 assays. The silencing efficiency of SOX18 in PCa cell lines was confirmed by western blotting (Fig. 2A). The results revealed that the cell proliferation of both cell lines transfected with siRNAs was significantly impaired compared with those transfected with siNC (Fig. 2B).

Knockdown of SOX18 notably suppresses the migration of PCa cells. To investigate the impact of SOX18 on PCa cell migration, Transwell assays were conducted with the same number of PC3 and DU145 cells transfected with siRNAs. Compared with their respective controls, knockdown of SOX18 notably suppressed the number of cells that crossed over the filter (Fig. 3).

Downregulation of SOX18 reduces the invasion ability of $\mathrm{PCa}$ cells. After establishing the role of SOX18 in the migration of PCa cells, we aimed to explore whether SOX18 is involved in the invasion of $\mathrm{PCa}$ cells. Therefore, we performed invasion assays using the same number of PC3 and DU145 cells transfected with siRNAs and siNC. As a result, downregulation of SOX18 significantly decreased the number of invaded cells compared with the controls (Fig. 4).

Knockdown of SOX18 suppresses tumor growth in nude mice. After the demonstration of the proliferation inhibition of SOX18 silencing in vitro, we subsequently used xenograft models in nude mice to investigate whether SOX18 silencing inhibited tumor growth in vivo. The same number of PC3 and DU145 cells transfected with LV-SOX18 or LV-NC were injected into each group of nude mice. The efficiency of SOX18 silencing was detected by western blotting (Fig. 5A). The tumor volume and weight in mice receiving cells transfected with LV-SOX18 were significantly decreased than those transfected with LV-NC (Fig. 5B and C).

SOX18 regulates the expression of TCF1, $c-M y c$, cyclin DI and MMP-7. To explore the molecular mechanisms of SOX18 underlying the proliferation, migration and invasion in $\mathrm{PCa}$ cells, we first assessed using western blotting several proteins 


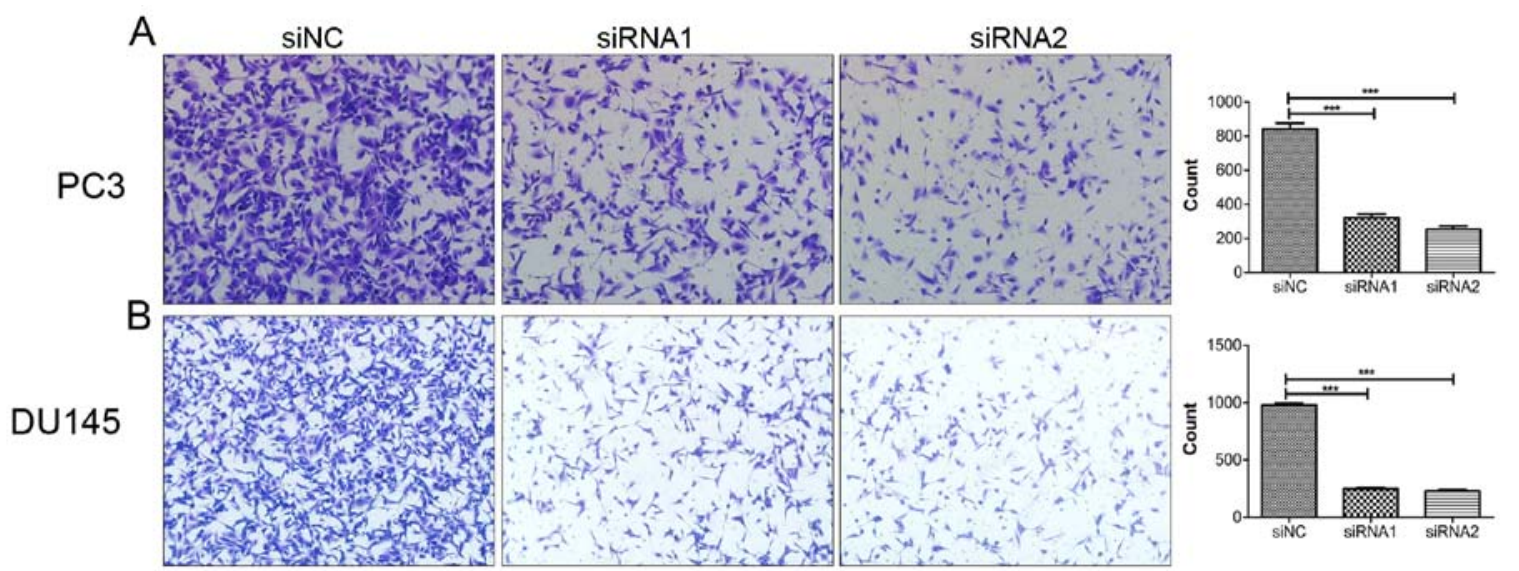

Figure 4. The invasion ability of prostate cancer cells was also reduced after SOX18 silencing. Compared with the siNC groups, the invaded (A) PC3 and (B) DU145 cells in the siRNA groups were significantly decreased $\left({ }^{* * *} \mathrm{P}<0.001\right)$.

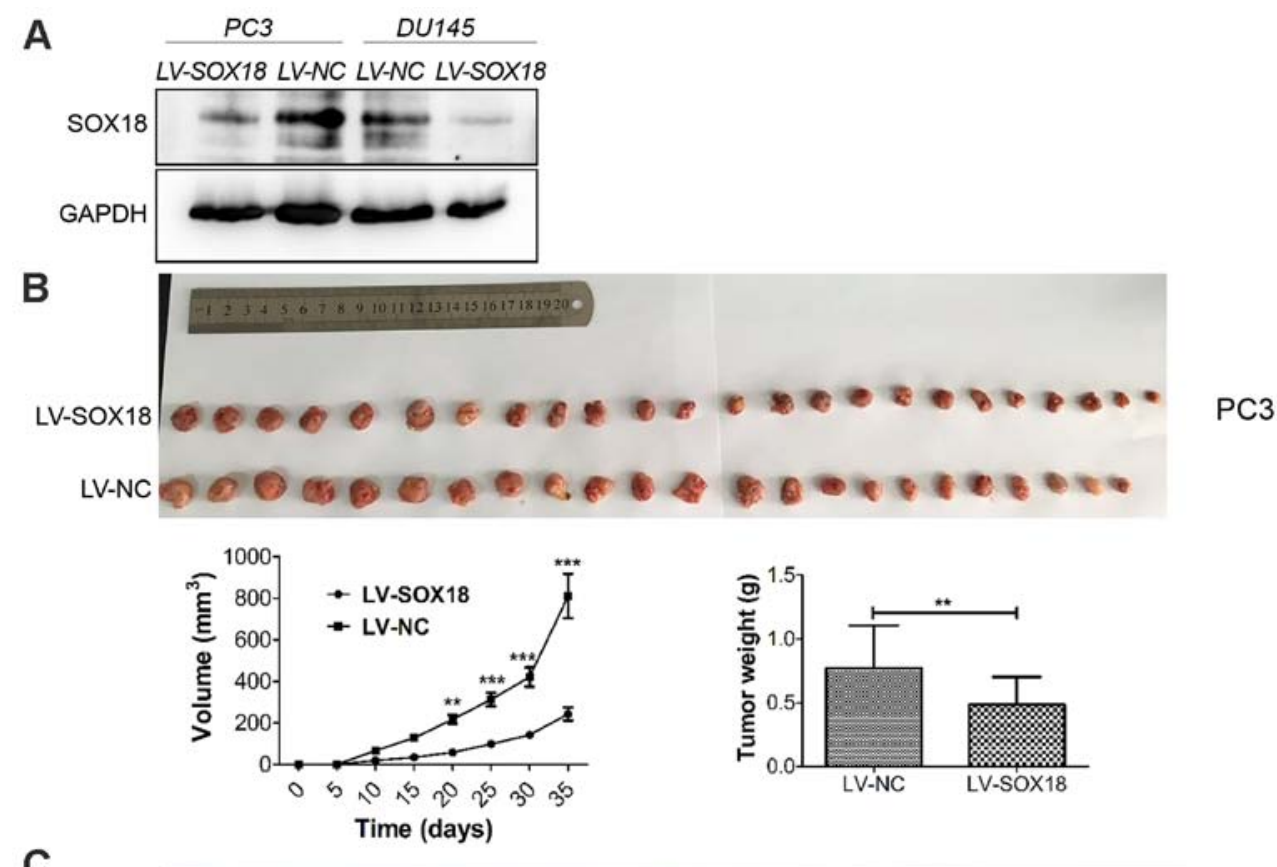

C
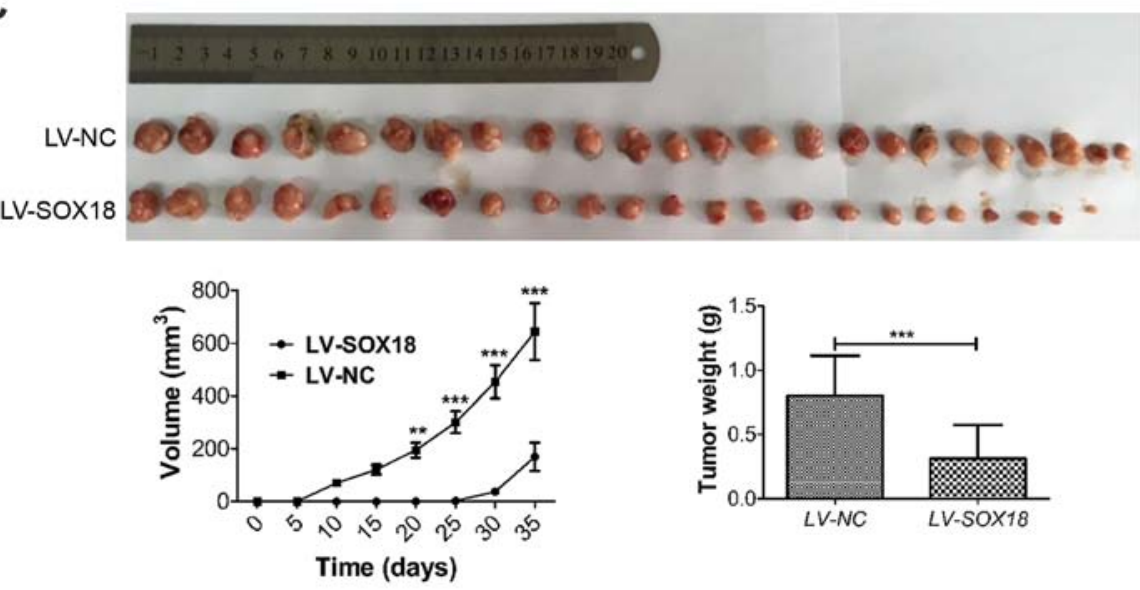

Figure 5. Silencing of SOX18 in prostate cancer cells suppresses tumor growth in nude mice. (A) The efficiency of SOX18 silencing in the PC3 and DU145 cells after transfection with the lentivirus was detected by western blotting. (B) The tumor volume and tumor weight were significantly decreased in the LV-SOX18 PC3 group. (C) The tumor volume and weight were also decreased in the LV-SOX18 DU145 group $\left.{ }^{(* *} \mathrm{P}<0.01,{ }^{* * *} \mathrm{P}<0.001\right)$.

that may be involved in the progression of PCa in PC3 cells after a 48 -h transfection with siRNAs and siNC. There were notably decreased protein levels of TCF1, c-Myc, cyclin D1 and MMP-7 in the SOX18 silenced cells compared with the 


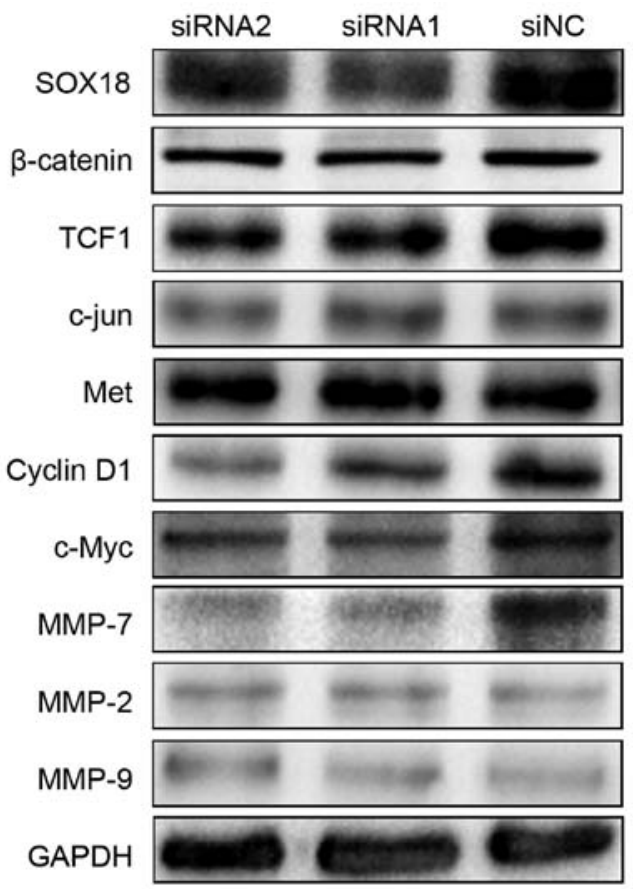

Figure 6. The expression of several proteins after SOX18 silencing in the PC3 cells. Compared with the siNC group, the expression levels of TCF1, c-Myc, cyclin D1 and MMP-7 were decreased in the siRNA groups. There were no differences in the expression of $\beta$-catenin, c-jun, Met, MMP-2 and MMP-9 between siRNAs and siNC lanes.

controls. Considering these signals are involved in the Wnt signaling pathway, we detected several other Wnt family members (including $\beta$-catenin, c-Jun and Met), but found no differences. In addition, we detected two other members of the MMP family (MMP-2 and MMP-9), but also found no differences (Fig. 6).

\section{Discussion}

Recently, a growing number of studies suggest that SOX18 is overexpressed in various types of tumors, and plays an important role in tumor occurrence and progression (13). The role and the potential function of SOX18 in various types of cancer have been revealed. Since the SOX18 gene behaves as an oncogene in various types of human cancer, its targeting has great therapeutic potential. In the present study, we found that SOX18 was significantly overexpressed in prostate cancer (PCa) tissues using immunohistochemistry, which was further confirmed with the expression of SOX18 at both the mRNA and protein level by western blotting and RT-PCR. A high expression of SOX18 was related to poor clinical characteristics (including a higher Gleason score, a higher histological grade and an advanced clinical stage) of patients with PCa. In addition, SOX18 was confirmed to be involved in the proliferation, migration and invasion of $\mathrm{PCa}$ cell lines in vitro and involved in tumor growth in vivo. As for the mechanisms of SOX18 underlying the progression of $\mathrm{PCa}$, we found that TCF1, c-Myc, cyclin D1 and MMP-7 were decreased when SOX18 was silenced by siRNAs in the PC 3 cell line. Our data indicated that SOX18 may be of diagnostic and therapeutic value for $\mathrm{PCa}$.
Previous studies demonstrated that SOX18 promoted the cell proliferation of HepG2 hepatocellular carcinoma (HCC) and MCF-7 breast cancer cells $(15,17)$. Consistent with these findings, knockdown of SOX18 in PCa cell lines notably suppressed cell growth in vitro. Moreover, the role of SOX18 silencing in the suppression of cell proliferation was also confirmed in vivo in the present study. Notably, in vivo, the tumor size was almost equal to that of the control group, but inside the tumor, the tumor was full of the hydrops, which indicated that knockdown of SOX18 promoted apoptosis in the PCa cells (data not shown). In a study of SOX18 in HCC, SOX18 knockdown was found to induce G1 phase arrest and apoptosis of HCC cells, indicating that SOX18 may contribute to cell proliferation via the promotion of cell cycle progression from the $\mathrm{G} 1$ to the $\mathrm{S}$ phase and the suppression of apoptosis. In the present study, we discovered that cyclin D1 was reduced in the SOX18-knockdown cells at the protein level. Cyclin D1, a transcription factor, is associated with cell proliferation via the promotion of the cell cycle from G1 to the $\mathrm{S}$ transition $(22,23)$. Previous studies found that cyclin D1 is overexpressed and contributes to the androgen-dependent DNA damage repair in PCa cells (24). Although we had a lack of data from the trials to address the changes in the PCa cell cycle after SOX18 silencing, the decreased level of cyclin D1 expression caused by SOX18 silencing indicated that SOX18 may promote cell cycle transition from the G1 to the $\mathrm{S}$ phase via the regulation of the expression of cyclin D1 to accelerate the aggressiveness of PCa.

Matrix metalloproteinases (MMPs), a family of transcription factors, can regulate the tumor microenvironment mainly through the degradation of the extracelluar matrix, and were found to be increased in expression and activation in almost all human types of cancer including PCa compared with normal tissues. The upregulation of MMPs was found to be related to the enhanced invasion ability of PCa cells in vitro (25). Consistent with previous research, our data showed that SOX18 silencing also impaired the migration and invasion abilities of PCa cells. Markedly, we found a decreased protein level of MMP-7 in the SOX18-silenced PCa cells. Grindel et al reported that MMP-7 acts as a switch altering PCa cell behavior and favoring cell dispersion and invasiveness (26). MMP-2 and MMP-9 are also involved in the mobility of PCa (27), but our results showed no differences when comparing the siRNA and siNC groups. Hoeth et al reported that SOX18 regulated the expression of MMP-7 in human endothelial cells by directly combining to the promoter of MMP-7 and activating its transcription (19). Although our results did not confirm whether SOX18 could combine to the promoter of MMP-7, we did demonstrate that SOX18 may regulate the mobility of PC 3 cells via the regulation of MMP-7, but not that of MMP-2 or MMP-9. Whether or not the process of SOX18 regulation of MMP-7 in PCa is roughly analogous to that in endothelial cells needs further study.

However, cyclin D1, MMP-7, TCF1 and c-Myc also exhibited a decreased protein level in the SOX18-silencing cells. TCF1 and c-Myc are also transcriptional factors, which are involved in tumor progression (28). Cyclin D1, MMP-7, TCF1 and c-Myc were also found to be involved in the Wnt signaling pathway, which is an important pathway in tumorigenesis and tumor progression (29), and TCF1 is located in 
the upstream of c-Myc, cyclin D1 and MMP-7. We detected other Wnt members, but found no differences among the siRNA and siNC groups. Previous research has reported that SOX7 decreases the expression of c-Myc and cyclin D1 via the downregulation of $\mathrm{Wnt} / \beta$-catenin transcription through the HMG-box which is the common domain of all SOX family members (30). Whether a simple link between SOX18 and the Wnt signaling pathway exists warrants further study.

The research on transcription factors and signaling pathways related with cancer has gradually become a 'hot spot' in the field of cancer research. Controlling the expression levels of certain transcription factors or some key points in signaling pathways to regulate the epofenetic characteristics of cells are promising therapeutic approaches. Transcription factor SOX18 is overexpressed in PCa, and the expression of SOX18 is notably correlated with both the clinical characteristics of patients and the malignant biological behavior of PCa cells. SOX18 may promote PCa progression via the upregulation of various transcription factors, such as TCF1, c-Myc, cyclin D1 and MMP-7. Therefore, further research on SOX18 is of potential value for the early diagnosis, risk evaluation and therapeutic approaches of PCa.

\section{Acknowledgements}

We thank Professor Gongwei Wang and Chenglong Zhao (Department of Pathology, Peking University People's Hospital) for their technological support in the immunohistochemistry and staining evaluation.

\section{References}

1. Siegel RL, Miller KD and Jemal A: Cancer statistics, 2016. CA Cancer J Clin 66: 7-30, 2016.

2. Cuzick J, Thorat MA, Andriole G, Brawley OW, Brown PH, Culig Z, Eeles RA, Ford LG, Hamdy FC, Holmberg L, et al: Prevention and early detection of prostate cancer. Lancet Oncol 15: e484-e492, 2014.

3. Heidenreich A, Bastian PJ, Bellmunt J, Bolla M, Joniau S, van der Kwast T, Mason M, Matveev V, Wiegel T, Zattoni F, et al; European Association of Urology: EAU guidelines on prostate cancer. Part 1: Screening, diagnosis, and local treatment with curative intent-update 2013. Eur Urol 65: 124-137, 2014.

4. Sarkar A and Hochedlinger K: The sox family of transcription factors: Versatile regulators of stem and progenitor cell fate. Cell Stem Cell 12: 15-30, 2013.

5. She ZY and Yang WX: SOX family transcription factors involved in diverse cellular events during development. Eur J Cell Biol 94 547-563, 2015.

6. Castillo SD and Sanchez-Cespedes M: The SOX family of genes in cancer development: Biological relevance and opportunities for therapy. Expert Opin Ther Targets 16: 903-919, 2012.

7. Thu KL, Becker-Santos DD, Radulovich N, Pikor LA, Lam WL and Tsao MS: SOX15 and other SOX family members are important mediators of tumorigenesis in multiple cancer types. Oncoscience 1: 326-335, 2014

8. Dai W, Tan X, Sun C and Zhou Q: High expression of SOX2 is associated with poor prognosis in patients with salivary gland adenoid cystic carcinoma. Int J Mol Sci 15: 8393-8406, 2014

9. Shao W, Chen H and He J: The role of SOX-2 on the survival of patients with non-small cell lung cancer. J Thorac Dis 7: 1113-1118, 2015.

10. Zhou D, Bai F, Zhang X, Hu M, Zhao G, Zhao Z and Liu R: SOX10 is a novel oncogene in hepatocellular carcinoma through Wnt/ $\beta$-catenin/TCF4 cascade. Tumour Biol 35: 9935-9940, 2014

11. François M, Caprini A, Hosking B, Orsenigo F, Wilhelm D, Browne C, Paavonen K, Karnezis T, Shayan R, Downes M, et al: Sox18 induces development of the lymphatic vasculature in mice. Nature 456: 643-647, 2008.
12. Duong T, Koltowska K, Pichol-Thievend C, Le Guen L, Fontaine F, Smith KA, Truong V, Skoczylas R, Stacker SA, Achen MG, et al: VEGFD regulates blood vascular development by modulating SOX18 activity. Blood 123: 1102-1112, 2014

13. Wünnemann F, Kokta V, Leclerc S, Thibeault M, McCuaig C, Hatami A, Stheneur C, Grenier JC, Awadalla P, Mitchell GA, et al: Aortic dilatation associated with a de novo mutation in the SOX18 gene: Expanding the clinical spectrum of hypotrichosis-lymphedema-telangiectasia syndrome. Can J Cardiol 32: 135.e1-135.e7, 2016.

14. Saitoh T and Katoh M: Expression of human SOX18 in normal tissues and tumors. Int J Mol Med 10: 339-344, 2002.

15. Wang G, Wei Z, Jia H, Zhao W, Yang G and Zhao H: Knockdown of SOX18 inhibits the proliferation, migration and invasion of hepatocellular carcinoma cells. Oncol Rep 34: 1121-1128, 2015.

16. Pula B, Kobierzycki C, Solinski D, Olbromski M, NowakMarkwitz E, Spaczynski M, Kedzia W, Zabel M and Dziegiel P: SOX18 expression predicts response to platinum-based chemotherapy in ovarian cancer. Anticancer Res 34: 4029-4037, 2014.

17. Pula B, Olbromski M, Wojnar A, Gomulkiewicz A, Witkiewicz W, Ugorski M, Dziegiel P and Podhorska-Okolow M: Impact of SOX18 expression in cancer cells and vessels on the outcome of invasive ductal breast carcinoma. Cell Oncol 36: 469-483, 2013.

18. Petrovic I, Milivojevic M, Popovic J, Schwirtlich M, Rankovic B and Stevanovic M: SOX18 is a novel target gene of Hedgehog signaling in cervical carcinoma cell lines. PLoS One 10: e0143591, 2015.

19. Hoeth M, Niederleithner H, Hofer-Warbinek R, Bilban M, Mayer H, Resch U, Lemberger C, Wagner O, Hofer E, Petzelbauer $\mathrm{P}$, et al: The transcription factor SOX18 regulates the expression of matrix metalloproteinase 7 and guidance molecules in human endothelial cells. PLoS One 7: e30982, 2012.

20. Fontijn RD, Volger OL, Fledderus JO, Reijerkerk A, de Vries HE and Horrevoets AJ: SOX-18 controls endothelial-specific claudin-5 gene expression and barrier function. Am J Physiol Heart Circ Physiol 294: H891-H900, 2008.

21. Sheng Z, Liu Y, Qin C, Liu Z, Yuan Y, Hu F, Du Y, Yin H, Qiu X and $\mathrm{Xu} \mathrm{T}$ : IgG is involved in the migration and invasion of clear cell renal cell carcinoma. J Clin Pathol 69: 497-504, 2016.

22. Tu K, Liu Z, Yao B, Xue Y, Xu M, Dou C, Yin G and Wang J: BCL-3 promotes the tumor growth of hepatocellular carcinoma by regulating cell proliferation and the cell cycle through cyclin D1. Oncol Rep 35: 2382-2390, 2016.

23. Lee HR, Mitra J, Lee S, Gao SJ, Oh TK, Kim MH, Ha T and Jung JU: Kaposi's sarcoma-associated herpesvirus viral interferon regulatory factor 4 (vIRF4) perturbs the $\mathrm{G}_{1}-\mathrm{S}$ cell cycle progression via deregulation of the cyclin Dl gene. J Virol 90: $1139-1143,2015$.

24. Casimiro MC, Di Sante G, Ju X, Li Z, Chen K, Crosariol M, Yaman I, Gormley M, Meng H, Lisanti MP, et al: Cyclin D1 promotes androgen-dependent DNA damage repair in prostate cancer cells. Cancer Res 76: 329-338, 2016.

25. Zhang Y, Gong LH, Zhang HQ, Du Q, You JF, Tian XX and Fang WG: Extracellular ATP enhances in vitro invasion of prostate cancer cells by activating Rho GTPase and upregulating MMPs expression. Cancer Lett 293: 189-197, 2010.

26. Grindel BJ, Martinez JR, Pennington CL, Muldoon M, Stave J, Chung LW and Farach-Carson MC: Matrilysin/matrix metalloproteinase-7 (MMP7) cleavage of perlecan/HSPG2 creates a molecular switch to alter prostate cancer cell behavior. Matrix Biol 36: 64-76, 2014.

27. Kato T, Fujita Y, Nakane K, Mizutani K, Terazawa R, Ehara H, Kanimoto Y, Kojima T, Nozawa Y, Deguchi T, et al: CCR1/CCL5 interaction promotes invasion of taxane-resistant PC3 prostate cancer cells by increasing secretion of MMPs 2/9 and by activating ERK and Rac signaling. Cytokine 64: 251-257, 2013.

28. Fan L, Peng G, Sahgal N, Fazli L, Gleave M, Zhang Y, Hussain A and Qi J: Regulation of c-Myc expression by the histone demethylase JMJD1A is essential for prostate cancer cell growth and survival. Oncogene 35: 2441-2452, 2016.

29. Mohammed MK, Shao C, Wang J, Wei Q, Wang X, Collier Z, Tang S, Liu H, Zhang F, Huang J, et al: Wnt $/ \beta$-catenin signaling plays an ever-expanding role in stem cell self-renewal, tumorigenesis and cancer chemoresistance. Genes Dis 3: 11-40, 2016.

30. Zhao T, Yang H, Tian Y, Xie Q, Lu Y, Wang Y, Su N, Dong B, Liu X, Wang C, et al: SOX7 is associated with the suppression of human glioma by HMG-box dependent regulation of Wnt/ $\beta$-catenin signaling. Cancer Lett 375: 100-107, 2016. 